\title{
3-D filter SQP method for optimal control of the multistage differential-algebraic systems with inconsistent initial values
}

\author{
Paweł Dra̧g \\ Institute of Computer Engineering, Control and Robotics \\ Wrocław University of Technology \\ Janiszewskiego 11-17, 50-372 Wrocław, Poland \\ Email: pawel.drag@pwr.edu.pl
}

\author{
Krystyn Styczeń \\ Institute of Computer Engineering, Control and Robotics, \\ Wrocław University of Technology \\ Janiszewskiego 11-17, 50-372 Wrocław, Poland \\ Email: krystyn.styczen@pwr.edu.pl
}

\begin{abstract}
In the article a 3-dimensional filter method for solving optimal control problems of differential-algebraic equations (DAEs) was presented. Direct multiple shooting method, which is appropriate for the control problems of the multistage DAE systems, leads to the large-scale nonlinear programming problems. In the proposed approach the extended Fletcher's filter with three inputs was used. The filter method promotes global convergence without the need to use a penalty function. The first input of the filter denotes the value of the cost function. The second and third inputs come from two types of equality constraints - consistent initial conditions of the DAE system and continuity constraints on the state trajectories. The new algorithm was tested on the optimal control problem of a fed-batch fermentor for penicillin production. The numerical simulations were executed in MATLAB environment using Wroclaw Center for Networking and Supercomputing.
\end{abstract}

Keywords-optimal control, DAE systems, inconsistent initial conditions, filter algorithm, nonlinear programming.

\section{INTRODUCTION}

C ONTROL and optimization of the complex and multistage differential-algebraic systems (DAEs) play a key role in a lot of technological systems. The dynamical behavior of the processes can be described by the differential equations. But conservation laws, balance equations, boundary conditions as well as interface with the environmental signals are modeled using the algebraic equations. Today, the differential-algebraic equations are one of the most elegant and simple ways to model a physical system, because they allow the creation of separate models for subcomponents that can then be pasted together [3], [4].

After some elimination processes, DAE systems can be rewritten in the form of ordinary differential equations, which can not present the nature of the process in the same manner like DAEs. A few advantages of a DAE formulation are the following: (1) it may be difficult to reformulate the problem as an ODE when nonlinearities are present, (2) the algebraic equations typically describe conservation laws or explicit equality constraints and they should be kept invariant, (3) it is easier to vary design parameters in an implicit model, (4) the implicit model does not require the modeling simplifications often necessary to get an ODE, (5) the variable keep their original physical interpretation, (6) the system structure can be exploited by problem-specific solvers, (7) less specialized mathematical expertise is required on the part of the designer [5].

One group of the approaches to the optimal control of complex dynamical processes are direct methods, which reformulate the original infinite dimensional optimization problem as a finite dimensional nonlinear programming (NLP) problem. In direct methods, the control and both differential and algebraic states are parametrized. Direct multiple shooting is one of the most popular direct methods. It enables using the efficient DAE solvers to calculate the function values and derivatives accurately. Since the integrations are decoupled on different multiple shooting intervals, this method is well suited for parallel computing. In this manner the control and optimization of the unstable dynamical modes can be considered. The approach allows an effective treatment of control and state path constraints [7]. Using the multiple shooting methods results in a large-scale NLP problem.

In this paper a tri-dimensional filter method based on the line search technique is considered. The filter method to solve nonlinear programming problem can be seen as an alternative to the traditional merit function approach. In this method, compared to the traditional penalty function methods, in which adjustment of the penalty parameter can be problematic, may make the trial steps accepted more easily.

The first idea of the filter method was to interpret the NLP problem as a bi-objective optimization problem with two conflicting purposes. The objective function had to be minimized, but the constraint violation should be minimized too. In the method presented in [10], all the constraints violations were added together and only one constraint violation was defined.

However, each constraint may have its own behavior. Some constraints can be highly nonlinear, while some others are linear or nearly linear [17]. There is the other situation, when the constraints can be grouped depending on the role in the mathematical model.

In the article the constraints were splited into equality constraints for consistent initial conditions for DAE model and equality constraints, which measure the discontinuity of the differential state trajectories. Thus the filter consists of three values: value of the objective function, equality constraints, 
which measure inconsistency of the initial conditions of DAE model, and equality constraints for continuity of the differential state trajectories.

The article is constructed as follows. In the 2nd section the multistage optimal control problem of differential-algebraic systems was presented. Then, in 3rd section, the 3-D filter algorithm was presented. The results of the numerical simulations were discussed in the 4th section.

In the article was used the same notation as in [7] and [20].

\section{PROBLEM STATEMENT}

Let us consider the problem of optimal control of the process with the performance cost function

$$
\min _{(u(t), x(t), z(t), p)} \int_{t_{0}}^{t_{f}} L(x(t), z(t), u(t), p) d t+E\left(x\left(t_{f}\right)\right),
$$

subject to a system of the index-one differential-algebraic equations (DAE) [6]

$$
\begin{aligned}
B(\cdot) \dot{x}(t) & =f(x(t), z(t), u(t), p) \\
0 & =g(x(t), z(t), u(t), p),
\end{aligned}
$$

where $x$ and $z$ denote the differential and algebraic state variables, respectively, $u$ is the vector valued control function, whereas $p$ is a vector of system parameters, which does not depend on the time. Matrix $B(x(t), z(t), u(t), p)$ is assumed to be invertible. Then the DAE is in a semi-explicit form.

The initial values for the differential and algebraic states and values for the system parameters are prescribed

$$
\begin{aligned}
& x\left(t_{0}\right)=x_{0}, \\
& p\left(t_{0}\right)=p_{0} .
\end{aligned}
$$

In addition, the terminal constraints

$$
r_{1}\left(x\left(t_{f}\right), p\right)=0, \quad r_{2}\left(x\left(t_{f}\right), p\right) \geq 0,
$$

as well as the state and control inequality constraints

$$
h(x(t), z(t), u(t), p) \geq 0
$$

have to be satisfied.

There is a quite other situation, when the multistage DAE system is considered, because each stage can be described by other set of the differential-algebraic equations.

Let us assume, that there are $N$ stages in the complex industrial process and there is an independent variable $t$, for example time or length of the chemical reactor.

For a suitable partition of the time horizon $\left[t_{0}, t_{f}\right]$ into $N$ subintervals $\left[t_{i}, t_{i+1}\right]$ with

$$
t_{0}<t_{1}<\cdots<t_{N}=t_{f}
$$

the control function $u(t)$ is discretized. It could be represented by a piecewise constant, piecewise linear or a polynomial approximation [19]. If the control function is parametrized as a piecewise constant vector function, then

$$
u(t)=u^{l}
$$

for $t \in\left[t_{l-1}, t_{l}\right], \quad l=1, \cdots, N$.
By the multiple shooting method, the DAE is parametrized in some sense too. The solution of the DAE system is decoupled on the $N$ intervals $\left[t_{l}, t_{l+1}\right]$. In this manner it introduces the initial values $s_{x}^{l}$ and $s_{z}^{l}$ of the differential and algebraic states at times $t_{i}$ as the additional optimization variables.

The trajectories $x(t)$ and $z(t)$ are obtained as a sum of trajectories $x^{l}(t)$ and $z^{l}(t)$ on each interval $\left[t_{l-1}, t_{l}\right]$. The trajectories $x^{l}(t)$ and $z^{l}(t)$ are the solutions of an initial value problem

$$
\begin{aligned}
B^{l}(\cdot) \dot{x}(t) & =f^{l}\left(x^{l}(t), z^{l}(t), u^{l}(t), p\right) \\
0 & =g^{l}\left(x^{l}(t), z^{l}(t), u^{l}(t), p\right)+\alpha^{l}\left(t_{l}\right) g^{l}\left(s_{x}^{l}, s_{z}^{l}, u^{l}, p\right) \\
t & \in\left[t_{l-1}, t_{l}\right], \quad l=1, \cdots, N .
\end{aligned}
$$

The relaxation parameter $\alpha^{l}\left(t_{l}\right)$ was introduced to allow an efficient DAE solution for the initial values and controls $s_{x}^{l}, s_{z}^{l}, u^{l}$, that may temporarily violate the consistency conditions. In this manner, the trajectories $x^{l}(t)$ and $z^{l}(t)$ on the interval $\left[t_{l-1}, t_{l}\right]$ are functions of the initial values, controls and parameters $s_{x}^{l}, s_{z}^{l}, u^{l}, p$.

The integral part of the cost function is evaluated on each interval independently

$$
\begin{gathered}
\min \begin{array}{l}
s_{x}^{l}, s_{z}^{l}, u^{l}, p \quad \int_{t_{0}}^{t_{1}} L^{1}\left(x^{1}(t), z^{1}(t), u^{1}(t), p\right) d t+\cdots+ \\
l=1, \cdots, N
\end{array} \\
+\int_{t_{N-1}}^{t_{N}} L^{N}\left(x^{N}(t), z^{N}(t), u^{N}(t), p\right) d t+E\left(x\left(t_{N}\right)\right)= \\
=\min \begin{array}{c}
s_{x}^{l}, s_{z}^{l}, u^{l}, p \\
l=1, \cdots, N
\end{array} \\
\quad \sum_{l=1}^{N} \int_{t_{l-1}}^{t_{l}} L^{l}\left(x^{l}(t), z^{l}(t), u^{l}(t), p\right) d t+ \\
+E\left(x\left(t_{N}\right)\right) .
\end{gathered}
$$

The parametrization of the optimal control problem of the multistage DAE systems using the multiple shooting approach and a piecewise constant control representation leads to the following nonlinear programming problem

$$
\begin{array}{cc}
\min s_{x}^{l}, s_{z}^{l}, u^{l}, p & \sum_{l=1}^{N} \int_{t_{l-1}}^{t_{l}} L^{l}\left(x^{l}(t), z^{l}(t), u^{l}(t), p\right) d t+ \\
l=1, \cdots, N & \\
& +E\left(x\left(t_{N}\right)\right)=\min _{\chi} \Phi(\chi),
\end{array}
$$

subject to the continuity conditions

$$
s_{x}^{l}=x^{l-1}\left(t_{l-1}\right), \quad l=2, \cdots, N,
$$

the consistency conditions

$$
0=g^{l}\left(s_{x}^{l}, s_{z}^{l}, u^{l}, p\right), \quad l=1, \cdots, N,
$$

control and path constraints imposed pointwise at the multiple shooting nodes

$$
h^{l}\left(s_{x}^{l}, s_{z}^{l}, u^{l}, p\right) \geq 0, \quad l=1, \cdots, N,
$$

the terminal constraints

$$
r_{1}\left(s_{x}^{l}, s_{z}^{l}, p\right)=0, \quad r_{2}\left(s_{x}^{l}, s_{z}^{l}, p\right) \geq 0,
$$


lower and upper bounds on the decision variables

$$
\begin{gathered}
\chi_{L} \leq \chi \leq \chi_{U} \\
\chi=\left[s_{x}^{1}, \cdots, s_{x}^{N}, s_{z}^{1}, \cdots, s_{z}^{N}, u^{1}, \cdots, u^{N}, p\right]^{T}, \\
\chi_{L}=\left[s_{x, L}^{1}, \cdots, s_{x, L}^{N}, s_{z, L}^{1}, \cdots, s_{z, L}^{N}, u_{L}^{1}, \cdots, u_{L}^{N}, p_{L}\right]^{T} \\
\chi_{U}=\left[s_{x, U}^{1}, \cdots, s_{x, U}^{N}, s_{z, U}^{1}, \cdots, s_{z, U}^{N}, u_{U}^{1}, \cdots, u_{U}^{N}, p_{U}\right]^{T}
\end{gathered}
$$

and with the DAE system in each interval

$$
\begin{aligned}
B^{l}(\cdot) \dot{x}(t) & =f^{l}\left(x^{l}(t), z^{l}(t), u^{l}(t), p\right) \\
0 & =g^{l}\left(x^{l}(t), z^{l}(t), u^{l}(t), p\right)+\alpha^{l}\left(t_{l}\right) g^{l}\left(s_{x}^{l}, s_{z}^{l}, u^{l}, p\right), \\
t & \in\left[t_{l-1}, t_{l}\right], \quad l=1, \cdots, N .
\end{aligned}
$$

\section{THE FILTER METHOD}

About 10 years ago, Fletcher and Leyffer proposed the filter methods to solve nonlinear programming (NLP) as an alternative to the traditional merit function approach. The underlying concept of filter is quite simple, being based on the multiobjective optimization, that is, the trial point is accepted provided there is a sufficient decrease of the objective function or the constraint violation function. In addition, the computational results presented in [10] were also very encouraging. The trust region filter sequential quadratic programming (SQP) methods have been studied in [9], [11]. On the other hand, the filters approach has been used also in conjunction with the line search strategy [20], [21], with interior point methods [18] and with the pattern search method [1]. Finally, the multidimensional filters have been employed to solve least squares problems, nonlinear equations and unconstrained optimization problems [12], [13].

In this paper, a tri-dimensional filter method based on the line search strategy, was proposed. The main idea of a filter is to interpret the NLP problem as a bi-objective optimization problem with two conflicting purposes: minimizing the objective function and the constraints violation. So, the formal filter in [10] consisted of two parts: the value of the objective function and the constraint violation. It means, that all the constraints are considered together and only one constraint violation is defined. However, each constraint may have its own behavior. For example, some constraints may be highly nonlinear, while some others are linear or nearly linear. In this work only equality constraints are considered. But they are two kinds of constraints, which have definitely a different meaning and applications. Thus, the new filter consists of three inputs: objective function value, inconsistency of the initial conditions for differential-algebraic equations and continuity of the differential state trajectories.

\section{A. A line search filter approach}

As it was assumed, the considered problem is stated as

$$
\min _{\chi \in \mathcal{R}^{n}} f(\chi)
$$

subject to

$$
c(\chi)=0,
$$

where the objective function $f: \mathcal{R}^{n} \rightarrow \mathcal{R}$ and the equality constraints $c: \mathcal{R}^{n} \rightarrow \mathcal{R}^{m}$ with $m<n$ are sufficiently smooth.
The Karush-Kuhn-Tucker (KKT) for the nonlinear programming problem (21)-(22) are

$$
\begin{gathered}
g(\chi)+A(\chi) \lambda=0, \\
c(\chi)=0,
\end{gathered}
$$

where $A(\chi)=\nabla c^{T}(\chi)$ denotes the transpose of the Jacobian of the constraints $c(\chi)$ and $g(\chi)=\nabla f(\chi)$ denotes the gradient of the objective function. The vector $\lambda$ corresponds to the Lagrange multipliers for the equality constraints. Under constraint qualifications assumption, the KKT conditions are the first order optimality conditions for (21)-(22) [15].

Given an initial estimate $\chi_{0}$, the line search algorithm generates a sequence of improved estimates $\chi_{k}$ of the solution for the NLP. For this purposes in each iteration $k$ a search direction $d_{k}$ is computed from the linearization at $\chi_{k}$ of the KKT conditions

$$
\left[\begin{array}{cc}
H_{k} & A_{k} \\
A_{k}^{T} & 0
\end{array}\right]\left(\begin{array}{c}
d_{k} \\
\delta \lambda_{k}
\end{array}\right)=-\left(\begin{array}{c}
g_{k} \\
c_{k}
\end{array}\right),
$$

where $A_{k}=A\left(\chi_{k}\right), g_{k}=g\left(\chi_{k}\right)$ and $c_{k}=c\left(\chi_{k}\right)$.

The symmetric matrix $H_{k}$ denotes the Hessian $\nabla_{\chi \chi}^{2} \mathcal{L}\left(\chi_{k}, \lambda_{k}\right)$ of the Lagrangian

$$
\mathcal{L}(\chi, \lambda)=f(\chi)+c^{T}(\chi) \lambda
$$

of the nonlinear programming problem or an approximation of the Hessian.

The vector $\lambda_{k}$ is some estimate of the optimal multipliers corresponding to the equality constraints, and $\delta \lambda_{k}$ in (25) can be used to determine a new estimate $\lambda_{k+1}$ for the next iteration.

After a search direction $d_{k}$ has been computed, a step size $\alpha_{k} \in(0,1]$ is determined in order to obtain the next iterate

$$
\chi_{k+1}=\chi_{k}+\alpha_{k} d_{k}
$$

It would be ideally to guarantee, that the sequence $\chi_{k}$ of iterates converges to a solution of the NLP. So, for this purposes, a backtracking procedure was proposed.

In the backtracking line search procedure a decreasing sequence of step size $\alpha_{k, l} \in(0,1] \quad(l=0,1,2, \cdots)$ is tried until some acceptance criterion is satisfied. Traditionally, a trial step size $\alpha_{k, l}$ is accepted if the corresponding trial point

$$
\chi_{k}\left(\alpha_{k, l}\right)=\chi_{k}+\alpha_{k, l} d_{k}
$$

provides sufficient reduction of a merit function, such as the exact penalty function

$$
\phi_{\rho}(\chi)=f(\chi)+\rho \theta(\chi),
$$

where the infeasibility measure $\theta(\chi)$ was defined as

$$
\theta(\chi)=\|c(\chi)\|
$$

Under certain regularity assumptions it can be shown that a feasible strict local minimum of the exact penalty function coincides with a local solution of the NLP if the value of the penalty parameter $\rho>0$ is chosen sufficiently large.

The overall algorithm for solving the equality constrained NLP problem is as follows. 


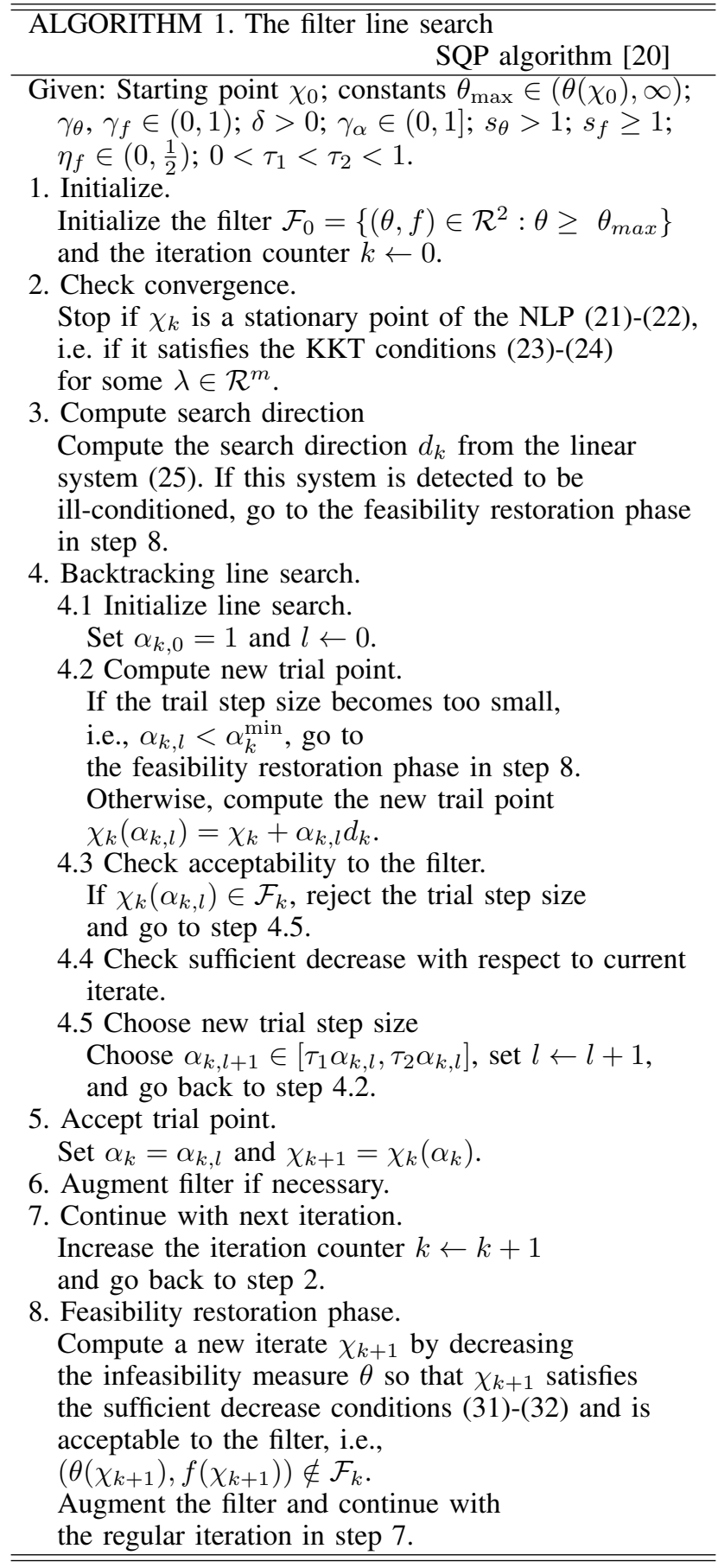

Line search methods that use a merit function ensure sufficient progress toward the solution. Hence, here it is required that the next iterate provides at least as much progress in one of the measures $\theta$ or $f$ that corresponds to a small fraction of the current constraint violation, $\theta\left(\chi_{k}\right)$. It means, that for fixed constants $\gamma_{\theta}, \gamma_{f} \in(0,1)$ a trial step size $\alpha_{k, l}$ provides sufficient reduction with respect to the current iterate $\chi_{k}$ if

$$
\theta\left(\chi_{k}\left(\alpha_{k, l}\right)\right) \leq\left(1-\gamma_{\theta}\right) \theta\left(\chi_{k}\right)
$$

or

$$
f\left(\chi_{k}\left(\alpha_{k, l}\right)\right) \leq f\left(\chi_{k}\right)-\gamma_{f} \theta\left(\chi_{k}\right)
$$

In a practical implementation, the constants $\gamma_{\theta}, \gamma_{f}$ typically are chosen to be small.

\section{B. A multidimensional filter}

The multidimensional filter algorithm was stated by Gould, Leyffer and Toint in the article [12]. It was used for solving nonlinear equations and nonlinear least-squares.

The following system of nonlinear equations is considered

$$
c(\chi)=0,
$$

where $c$ is twice continously differentiable function from $\mathcal{R}^{n}$ into $\mathcal{R}^{m}$. In the next step, the equation (33) is partitioned into $p$ sets $\left\{c_{i}(\chi)\right\}_{i \in \mathcal{I}_{j}}$ for $j=1, \cdots, p$, with $\{1, \cdots, n\}=$ $\mathcal{I}_{1} \cup \mathcal{I}_{2} \cup \cdots \mathcal{I}_{p}$ and

$$
\theta_{j}(\chi)=\left\|c_{\mathcal{I}_{j}}\right\|
$$

for $j=1, \cdots, p$, where $\|\cdot\|$ is the Euclidean norm and $c_{\mathcal{I}_{j}}$ is the vector whose components are the components of $c$ indexed by $\mathcal{I}_{j}$.

The point is therefore a solution of (33) if and only if

$$
\theta_{j}(\chi)=0
$$

for $j=1, \cdots, p$. The quantity $\theta_{j}(\chi)$ may be interpreted as the size of the residual of the $j$ th set of equations at the point $\chi$.

The classical approach for solving (33) is to minimize a merit function involving some norm of the residual

$$
\min _{\chi \in \mathcal{R}^{n}} f(\chi)=\frac{1}{2}\|\theta(\chi)\|^{2} .
$$

The main idea of filter algorithms for constrained optimization is that new iterates of the underlying iterative algorithm can be accepted if they do not perform, compared to past iterates kept in the filter, worse on both important and typically conflicting accounts for this type of problem: feasibility and low objective function value.

In the context of nonlinear equations, one may consider driving each of the $\left\{\theta_{i}(\chi)\right\}_{i=1}^{p}$ to zero as an independent task.

A point $\chi_{1}$ dominates a point $\chi_{2}$ whenever

$$
\forall j=1, \cdots, p \quad \theta_{j}\left(\chi_{1}\right) \leq \theta_{j}\left(\chi_{2}\right) .
$$

Thus, if iterate $\chi_{k_{1}}$ dominates iterate $\chi_{k_{2}}$, the latter is of no real interest, since $\chi_{k_{1}}$ is at least as good as $\chi_{k_{2}}$ for each of the equation sets. All, what is needed to do now, is to remember iterates that are not dominated by other iterates using a structure called a filter.

A filter is a list $\mathcal{F}$ of $p$-tuples of the form $\left(\theta_{1, k}, \cdots, \theta_{p, k}\right)$ such that

$$
\theta_{j, k}<\theta_{j, l}
$$

for at least one $j \in\{1, \cdots, p\}$ and $k \neq l$.

Filter methods propose to accept a new trial iterate $\chi_{k}^{+}$if it is not dominated by any other iterate in the filter and $\chi_{k}$.

Additionally, it is inappropriate to accept a new point $\chi_{k}^{+}$if $\theta\left(\chi_{k}\right)$ is arbitraily close to being dominated by another point 
already in the filter [16]. To avoid this situation, the acceptability condition should be more strength. More formally, one can say that a new trial point $\chi_{k}^{+}$is acceptable for the filter $\mathcal{F}$ if and only if

$\forall \theta_{l} \in \mathcal{F} \quad \exists j \in\{1, \cdots, p\} \quad \theta_{j}\left(\chi_{k}^{+}\right)<\theta_{j, l}-\gamma_{\theta} \delta\left(\left\|\theta_{l}\right\|,\left\|\theta_{k}^{+}\right\|\right)$,

where $\gamma_{\theta} \in(0,1 / \sqrt{p})$ is a small positive constant and where $\delta(\cdot, \cdot)$ is one of the following

$$
\begin{gathered}
\delta\left(\left\|\theta_{l}\right\|,\left\|\theta_{k}^{+}\right\|\right)=\left\|\theta_{l}\right\| \\
\delta\left(\left\|\theta_{l}\right\|,\left\|\theta_{k}^{+}\right\|\right)=\left\|\theta_{k}^{+}\right\|
\end{gathered}
$$

or

$$
\delta\left(\left\|\theta_{l}\right\|,\left\|\theta_{k}^{+}\right\|\right)=\min \left(\left\|\theta_{l}\right\|,\left\|\theta_{k}^{+}\right\|\right) .
$$

\section{A tri-dimensional filter SQP algorithm}

The groups of constraints violations for NLP problem (21)(22) are defined as follows

$$
\mathcal{S}_{I}=\frac{1}{2} \sum_{i=1}^{p} c_{i}^{2}(\chi)
$$

for continuity of the differential state trajectories and

$$
\mathcal{S}_{I I}=\frac{1}{2} \sum_{i=p+1}^{m} c_{i}^{2}(\chi),
$$

which represents inconsistency of the initial conditions.

These two groups of constraints are defined similarly, but they have definitely different meanings and play another role in the optimization process.

In a filter $\mathcal{F}$, triples of values $\left(\mathcal{S}_{I}(\chi), \mathcal{S}_{I I}(\chi), f(\chi)\right)$ are considered.

Definition 1. The iterate $\chi_{k}$ dominates the iterate $\chi_{l}$ if and only if $\mathcal{S}_{I}\left(\chi_{k}\right) \leq \mathcal{S}_{I}\left(\chi_{l}\right), \mathcal{S}_{I I}\left(\chi_{k}\right) \leq \mathcal{S}_{I I}\left(\chi_{l}\right)$ and $f\left(\chi_{k}\right) \leq$ $f\left(\chi_{l}\right)$. It is denoted by $\chi_{k} \preceq \chi_{l}$.

Thus, if $\chi_{k} \preceq \chi_{l}$, the latter is of no real interest, since $\chi_{k}$ is at least as good as $\chi_{l}$ with respect to three violations. Furthermore, if $\chi_{k} \preceq \chi_{l}$, one can say that the triple $\left(\mathcal{S}_{I}\left(\chi_{k}\right), \mathcal{S}_{I I}\left(\chi_{k}\right), f\left(\chi_{k}\right)\right)$ dominates the triple $\left(\mathcal{S}_{I}\left(\chi_{l}\right), \mathcal{S}_{I I}\left(\chi_{l}\right), f\left(\chi_{l}\right)\right)$

Definition 2. The $k$ th filter is a list of triples $\left.\left\{\mathcal{S}_{I}\left(\chi_{l}\right), \mathcal{S}_{I I}\left(\chi_{l}\right), f\left(\chi_{l}\right)\right)\right\}_{l<k}$, such that no triple dominates any other.

Let $\mathcal{F}_{k}$ denote the indices in the $k$ th filter

$$
\mathcal{F}_{k}=\left\{l<k: \chi_{j} \npreceq \chi_{l} \quad \forall j \in\{0,1,2, \cdots, k-1\} \backslash\{l\}\right\}
$$

Filter methods accept a trial point $\chi_{k+1}=\chi_{k}+\alpha d_{k}$ if its corresponding triple $\left(\mathcal{S}_{I}\left(\chi_{k+1}\right), \mathcal{S}_{I I}\left(\chi_{k+1}\right), f\left(\chi_{k+1}\right)\right)$ is not dominated by any other triple in the $k$ th filter, neither the triple corresponding to $\chi_{k}$, i.e. $\left(\mathcal{S}_{I}\left(\chi_{k}\right), \mathcal{S}_{I I}\left(\chi_{k}\right), f\left(\chi_{k}\right)\right)$

Definition 3. A new trial point $\chi_{k+1}$ is said to be "acceptable to the $k$ th filter" if $\chi_{k+1}$ is acceptable to $\chi_{l}$ for all $l \in \mathcal{F}_{k}$.

In this manner defined 3-dimensional line search-SQP filter was tested on optimal control problem of nonlinear differentialalgebraic system with inconsistent initial conditions.

\section{CASE STUDY: OPTIMAL CONTROL OF A FED-BATCH FERMENTOR FOR PENICILLIN PRODUCTION}

This problem considers a fed-batch reactor for the production of penicillin [2]. We consider here the free terminal time version where the objective is to maximize the amount of penicillin using the feed rate as the control variable. The mathematical statement of the free terminal time problem is as follows.

Find $u(t)$ and $t_{f}$ over $t \in\left[t_{0}, t_{f}\right]$ to maximize

$$
J=x_{2}\left(t_{f}\right) \cdot x_{4}\left(t_{f}\right)
$$

subject to differential-algebraic system

$$
\begin{gathered}
\frac{d x_{1}}{d t}=h_{1} x_{1}-u\left(\frac{x_{1}}{500 x_{4}}\right), \\
\frac{d x_{2}}{d t}=h_{2} x_{1}-0.01 x_{2}-u\left(\frac{x_{2}}{500 x_{4}}\right), \\
\frac{d x_{3}}{d t}=-h_{1} \frac{x_{1}}{0.47}-h_{2} \frac{x_{1}}{1.2}-x_{1} \frac{0.029 x_{3}}{0.0001+x_{3}}+\frac{u}{x_{4}}\left(1-\frac{x_{3}}{500}\right), \\
\frac{d x_{4}}{d t}=\frac{u}{500}, \\
h_{1}=0.11\left(\frac{x_{3}}{0.006 x_{1}+x_{3}}\right), \\
h_{2}=0.0055\left(\frac{x_{3}}{0.0001+x_{3}\left(1+10 x_{3}\right)}\right)
\end{gathered}
$$

where $x_{1}, x_{2}$ and $x_{3}$ are the biomass, penicillin and substrate concentration $(g / L)$, and $x_{4}$ is the volume $(L)$. The initial conditions are

$$
x\left(t_{0}\right)=\left[\begin{array}{llll}
1.5 & 0 & 0 & 7
\end{array}\right]^{T} .
$$

There are several path constraints for state variables

$$
\begin{aligned}
& 0 \leq x_{1} \leq 40 \\
& 0 \leq x_{2} \leq 25, \\
& 0 \leq x_{3} \leq 10 .
\end{aligned}
$$

The upper and lower bounds on the only control variable (feed rate of substrate) are

$$
0 \leq u \leq 50
$$

The control problem of a fed-batch fermentor for penicillin production was solved with the proposed 3-D SQP filter algorithm combined with multiple shooting method.

At first, the overall time domain was divided into 20 equidistant intervals. It results in 20 differential-algebraic submodels, each of them consists of 4 differential equations and 2 algebraic equations. Initial conditions only for the first stage are known. So, there are 76 decision variables connected with initial values for differential variables and 40 variables, which represent pointwise values of algebraic states. The last decision variable was the duration time of the process. 


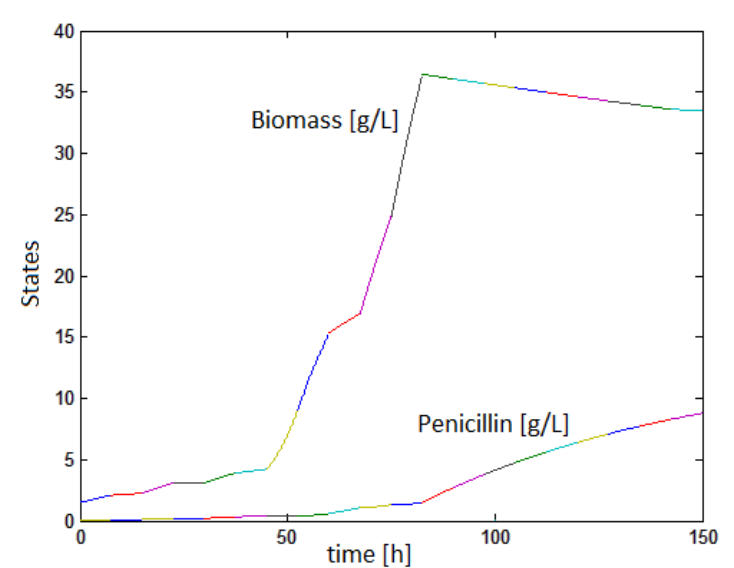

Fig. 1. The optimal trajectories of the both biomass and penicillin concentrations

Backward Differentiation Formula of order 1 was used to solve DAE systems at each stage.

The initial values for decision variables were as follows

$$
\begin{gathered}
\chi_{1, x_{1,2}}, \cdots, \chi_{19, x_{1,20}}=1.5, \\
\chi_{20, x_{2,2}}, \cdots, \chi_{38, x_{2,20}}=0.0, \\
\chi_{39, x_{3,2}}, \cdots, \chi_{57, x_{3,20}}=0.0, \\
\chi_{58, x_{4,2}}, \cdots, \chi_{76, x_{4,20}}=7.0, \\
\chi_{77, h_{1,1}}, \cdots, \chi_{96, h_{1,20}}=10.0, \\
\chi_{97, h_{2,1}}, \cdots, \chi_{116, h_{2,20}}=10.0, \\
\chi_{117, u_{1}}, \cdots, \chi_{136, u_{20}}=10.0, \\
\chi_{137, t_{f}}=110.0[h] .
\end{gathered}
$$

The solution, with the accuracy $10^{-6}$ for each input of the filter, was obtained after 6 hours. The final value of the objective function is $89.5473[\mathrm{~g}]$. The duration of the whole process is 150 hours. There are the optimal trajectories of the both biomass and penicillin concentrations in Fig. 1.

\section{CONCLUSION}

In the article optimal control problem of complex systems with differential-algebraic constraints was considered. One of the most important question in optimization and control of DAE systems concerns on consistent initial conditions. For these purposes the 3-dimensional line search-SQP filter was designed. It enables simultaneous optimization of objective function and treatment of constraints.

The filter algorithm, which consists of three inputs, was tested on the optimal control problem of a fed-batch fermentor for penicillin production. The encouraging results were obtained.

In real-life applications, very often multistage technological processes are under considerations. The multiple shooting method, which enables efficient treatment of such processes, incorporates additional equality constraints and decision variables to the NLP problem, connected with continuity of the differential state trajectories.

The most important advantage of the multiple shooting method is a possibility of control and optimization of highly nonlinear differential-algebraic systems in an open-loop. Application in this field is currently also an important challenge for the presented 3-dimensional SQP filter algorithm [8], [14].

\section{ACKNOWLEDGMENT}

The project was supported by the grant of National Science Centre Poland DEC- 2012/07/B/ST7/01216.

\section{REFERENCES}

[1] C. Audet, J.E. Dennis Jr. 2004. A pattern search filter method for nonlinear programming without derivatives. SIAM Journal on Optimization. 14:980-1010, http://dx.doi.org/10.1137/S105262340138983X.

[2] J.R. Banga, E. Balsa-Canto, C.G. Moles, A.A. Alonso. 2005. Dynamic optimization of bioprocesses: Efficient and robust numerical strategies. Journal of Biotechnology. 117:407-419, http://dx.doi.org/10.1016/j.jbiotec.2005.02.013.

[3] J.T. Betts. 2010. Practical Methods for Optimal Control and Estimation Using Nonlinear Programming, Second Edition. SIAM, Philadelphia, http://dx.doi.org/10.1137/1.9780898718577.

[4] L.T. Biegler. 2010. Nonlinear Programming. Concepts, Algorithms and Applications to Chemical Processes. SIAM, Philadelphia, http://dx.doi.org/10.1137/1.9780898719383.

[5] L.T. Bielger, S. Campbell, V. Mehrmann. 2012. DAEs, Control, and Optimization. Control and Optimization with Differential-Algebraic Constraints. SIAM, Philadelphia, http://dx.doi.org/10.1137/9781611972252.ch1.

[6] K.E. Brenan, S.L. Campbell, L.R. Petzold. 1996. Numerical Solution of Initial- Value Problems in Differential-Algebraic Equations. SIAM, Philadelphia, http://dx.doi.org/10.1137/1.9781611971224.

[7] M. Diehl, H.G. Bock, J.P. Schlöder, R. Findeisen, Z. Nagy, F. Allgower. 2002. Real-time optimization and nonlinear model predictive control of processes governed by differential-algebraic equations. Journal of Process Control. 12:577-585, http://dx.doi.org/10.1016/S0959-1524(01)00023-3

[8] P. Drạg, K. Styczeń. 2012. A Two-Step Approach for Optimal Control of Kinetic Batch Reactor with electroneutrality condition. Przegląd Elektrotechniczny. 6:176-180.

[9] R. Fletcher, N.I.M. Gould, S. Leyffer, P.L. Toint, A. Watcher. 2002. Global convergence of a trust region SQP-filter algorithms for general nonlinear programming. SIAM Journal on Optimization. 13:635-659, http://dx.doi.org/10.1137/S1052623499357258.

[10] R. Fletcher, S. Leyffer. 2002. Nonlinear programming without a penalty function. Mathematical Programming. 91:239-269, http://dx.doi.org/10.1007/s101070100244.

[11] R. Fletcher, S. Leyffer, P.L. Toint. 2002. On the global convergence of a Filter-SQP algorithm. SIAM Journal on Optimization. 13:44-59, http://dx.doi.org/10.1137/S105262340038081X.

[12] N.I.M. Gould, S. Leyffer, P.L. Toint. 2004. A multidimensional filter algorithm for nonlinear equations and nonlinear least squares. SIAM Journal on Optimization. 15:17-38, http://dx.doi.org/10.1137/S1052623403422637.

[13] N.I.M. Gould, C. Sainvitu, P.L. Toint. 2005. A filter trust region method for unconstrained optimization. SIAM Journal on Optimization. 16:341357, http://dx.doi.org/10.1137/040603851.

[14] M. Kwiatkowska. 2012. Antimicrobial PVC composites. Processing technologies and functional properties of polymer nanomaterials for food packaging : International COST Workshop, Wroclaw, Poland, September 11-12, 2012, pp. 40-41.

[15] J. Nocedal, S.J. Wright. 2006. Numerical Optimization. Second Edition. Springer, New York, http://dx.doi.org/10.1007/978-0-387-40065-5 
[16] E. Rafajłowicz, K. Styczeń, W. Rafajłowicz. 2012. A modified filter SQP method as a tool for optimal control of nonlinear systems with spatio-temporal dynamics. Int. J. Appl. Math. Comput. Sci. 22:313-326, http://dx.doi.org/10.2478/v10006-012-0023-8.

[17] C. Shen, W. Xue, D. Pu. 2009. Global convergence of a tri-dimensional filter SQP algorithm based on the line search method. Applied Numerical Mathematics. '59:235-250, http://dx.doi.org/10.1016/j.apnum.2008.01.005.

[18] M. Ulbrich, S. Ulbrich, L.N. Vicente. 2004. A globally convergent primal-dual interior filter method for nonconvex nonlinear programming. Mathematical Programming. 100:379-410, http://dx.doi.org/10.1007/s10107-003-0477-4.

[19] V.S Vassiliadis, R.W.H. Sargent, C.C. Pantelides. 1994. Solution of a Class of Multistage Dynamic Optimization Problems. 1. Problems without Path Constraints. Ind. Eng. Chem. Res. 33:2111-2122, http://dx.doi.org/10.1021/ie00033a014.

[20] A. Wätcher, L.T. Biegler. 2005. Line search filter methods for nonlinear programming: Motivation and global convergence. SIAM Journal on Optimization. 16: 1-31, http://dx.doi.org/10.1137/S1052623403426556.

[21] A. Wätcher, L.T. Biegler. 2005. Line search filter methods for nonlinear programming: Local convergence. SIAM Journal on Optimization. 16:3248, http://dx.doi.org/10.1137/S1052623403426544. 